1 Title: Adults and children blatantly dehumanize outgroups

2 Authors: Wen Zhou ${ }^{1}$, Brian Hare*1,2

3

4 Affiliations: ${ }^{1}$ Department of Evolutionary Anthropology, Duke University, Durham, NC, USA;

$5 \quad{ }^{2}$ Center for Cognitive Neuroscience, Duke University, Durham, NC, USA

6

7 Abstract: Dehumanization is observed in adults across cultures and is thought to motivate the

8 worst forms of human violence. The age of first expression and the degree of socialization

9 required to foster dehumanization remains largely untested. This research demonstrates that

10 several different representations of humanness, including a novel one, readily elicit blatant

11 dehumanization in adults $(\mathrm{N}=482)$ and children $(5-12$ years of age; $\mathrm{N}=150)$. We also find that

12 dehumanizing responses in both age groups are associated with stronger perception of outgroup

13 inferiority and a willingness to punish outgroup transgressions. These findings rule out the need

14 for exposure to cultural norms throughout adolescents and adulthood before observing

15 significant outgroup dehumanization.

16

17 Keywords: dehumanization, intergroup bias, moral concern, enculturation 
18 Dehumanization is a psychological process through which others are stripped of humanness.

19 Perceiving others as subhuman is posited to allow for moral exclusion. Heinous acts of cruelty

20 and violence become acceptable (Bandura, 2016; Smith, 2020). Dehumanization is linked to

21 acceptance of punishment, torture and capital punishment of outgroups (Bandura, Underwood, \&

22 Fromson, 1975; Jardina \& Piston, 2016; Kteily, Bruneau, Waytz, \& Cotterill, 2015; Markowitz

23 \& Slovic, 2020; Viki, Osgood, \& Phillips, 2013). In extreme cases, dehumanizing portrayals, for

24 example, of Jewish people as vermin during the Holocaust, Tutsis as cockroaches in the Rwanda

25 genocide, and victims as rats during the Tokyo firebombing are thought to have encouraged mass

26 killings. Dehumanization of enemies is used to encourage soldiers to apply lethal force (Smith,

27 2020). Understanding the mechanism that makes individuals predisposed to dehumanization and

28 the psychology that allows for its development are critical to implementing strategies to prevent

29 future intergroup aggression.

30 The Early Emergence hypothesis (EEH) posits that the tendency to deny humanity to

31 another individual or group emerges as result of a maturational pathway during early childhood

32 and develops little during formal schooling. This potential early maturation would support the

33 universality across humans which has been suggested by a range of findings. Small-scale

34 societies from across the world refer to their own group in language and myth as "principle" or

35 "real" people (McCallum, 2001; Sumner, 1906). Cross-cultural experimental surveys have

36 revealed blatant dehumanization of threatening outgroups in every ethnic and demographic

37 group surveyed (Kteily \& Bruneau, 2017). What is also prevalent across cultures is the

38 association of dehumanization with the perceived inferiority of outgroups (Kteily et al., 2015;

39 Trounson, Critchley, \& Pfeifer, 2015) and moral disengagement. The tendency of those surveyed

40 to dehumanize predicts their withdrawal of prosociality and the acceptance of harm toward other 
41 groups even when prejudice is controlled (Kteily \& Bruneau, 2017; Kteily et al., 2015; Kteily,

42 Hodson, \& Bruneau, 2016).

43 This early emergence of dehumanization is suggested to result in the maturation of the

44 cognitive process that allows for viewing another person or group of people as lacking some

45 combination of human perceptions, feelings, desires and beliefs (Hare, 2017; Hare \& Woods,

46 2020). The link between the Theory of Mind capacity and dehumanization is suggested by the

47 central role of mentalizing in promoting prosociality and inhibit antisocial behaviors to others

48 (Hare, 2017). Neurobiological evidence demonstrates mental states of threatening outgroup

49 members are not recognized (Bedny, Pascual-Leone, \& Saxe, 2009; Cikara, Bruneau, \& Saxe,

50 2011; Cikara, Eberhardt, \& Fiske, 2011; Harris \& Fiske, 2006; Levy et al., 2016; Richins,

51 Barreto, Karl, \& Lawrence, 2019; Viki et al., 2013). Blatant dehumanization shows neural

52 specificity that distinguishes it from negative or prejudice responses to others (Bruneau, Jacoby,

53 Kteily, \& Saxe, 2018). The left inferior parietal cortex (IPC) and left inferior frontal cortex (IFC)

54 selectively respond during blatant dehumanization - areas involved in reducing mentalization

55 and processing of social status (Bruneau et al., 2018; Cikara, Eberhardt, et al., 2011; Cloutier,

56 Ambady, Meagher, \& Gabrieli, 2012; Farrow et al., 2011; Jack, Dawson, \& Norr, 2013).

57 Consistent with the EEH, initial evidence suggests dehumanization of outgroups already occurs

58 in early childhood with the appearance of full-blown Theory-of-Mind abilities (McLoughlin,

59 Tipper, \& Over, 2018).

60 The Social Artifact hypothesis (SAH) offers an alternative to the EEH. It suggests

61 dehumanization results from enculturation. Through a lifetime of social learning and norm

62 enforcement, individuals learn to use non-human entities, especially animals, as metaphors to

63 emphasize negative traits of other groups (Dhont, Hodson, Loughnan, \& Amiot, 2019; Goff, 
64 Eberhardt, Williams, \& Jackson, 2008). While animal analogies are widely used to degrade

65 outgroups, the belief that humans are morally superior expresses itself late in development and is

66 thought to be largely socially acquired (Wilks, Caviola, Kahane, \& Bloom, 2020). Even the most

67 insulting animalistic slurs that engender disgust of other groups are only ever meant and

68 understood metaphorically (Over, 2020; Smith, 2020).

69 SAH points to a gap in evidence used to suggest EEH. It remains unknown what degree

70 of enculturation or training is required to observed dehumanization. Cross-cultural

71 questionnaires sample adults and have largely relied on a single measure of dehumanization

72 known as the Ascent of Man. This measure relies on the common misconception that evolution

73 in a linear progression toward human perfection (see Figure 1; Kteily \& Bruneau, 2017).

74 Similarly, children have only ranked humanness using the Face Continuum scale that requires a

75 degree of training (see Figure 1; McLoughlin et al, 2018). Another line of studies investigated

76 dehumanization using implicit and explicit trait attribution, and find that people attribute more

77 human-related qualities and secondary emotions to ingroup rather than outgroup targets (Haslam

78 \& Loughman, 2014). Because the trait attribution tasks require advanced language

79 comprehension abilities, only literate children were tested (Chas, Betancor, Delgado, \&

80 Rodríguez-Pérez, 2018; Costello \& Hodson, 2014; Martin, Bennett, \& Murray, 2008; van

81 Noorden, Haselager, Cillessen, \& Bukowski, 2014). Moreover, recent research reveals age

82 difference in associating emotion terms with humanness. As compared to literate children, adults

83 derive more clues about humanity from emotions which have cultural difference in expression

84 (Rodríguez-Pérez, Betancor-Rodríguez, Ariño-Mateo, Demoulin, \& Leyens, 2014; Rodríguez,

85 Villar, Rodríguez-Pérez, \& Rodríguez, 2016). Traits that are thought to represent humanness also

86 vary across cultures (Bain, Vaes, Kashima, Haslam, \& Guan, 2012). These findings suggest that 
87 the understanding of the association between traits and humanness is heavily influenced by

88 social learning. Together, the existing observations may only represent different participants'

89 idiosyncratic understanding and response to these specific representations of humanness.

90 Children may not spontaneously dehumanize without enculturation or explicit training by adults.

91 To test the two hypotheses, what is needed now is an assessment of dehumanizing

92 tendencies in children who have developed full-blown theory-of-mind (i.e. five years or older)

93 using multiple measures that vary in familiarity and intuitiveness. If dehumanization emerges

94 early $(\mathrm{EEH})$ with the maturation of Theory-of-Mind network, then a variety of stimuli that

95 provide a scale of humanness, regardless of familiarity, should illicit stronger dehumanizing

96 responses toward outgroup than ingroups. Children should be more likely to view the outgroup

97 as inferior while being less likely to act prosocially and more accepting of harm toward them.

98 This tendency will not change during middle childhood when children are exposed to formal

99 schooling. If dehumanization is largely a social artifact shaped by enculturation and familiarity

100 with materials (SAH), then each scale should illicit very different responses in children, and their

101 responses will largely be unassociated with the perception of group hierarchy, prosociality or

102 acceptance of harm. In pace with socialization, the dehumanizing tendency will develop with 103 age.

104

105 General Method

106 We used the Ascent of Man, Face Continuum, and Human Silhouette scales (see Figure 1) to 107 evaluate the tendency of school-age children to dehumanize outgroups. Each scale asked 108 participants to indicate how human a group is by using a scale from "the least human-like" to 109 "fully human". The Ascent of Man scale presents cartoons of human ancestors that are 
110 increasingly human-like and is validated for use with adults (Kteily et al., 2015). For use with

111 children, we adapted the scale by reducing it to four items and using more differentiated species

112 (i.e. those with more taxonomic distance between them). The Face Continuum scale was

113 previously developed for use with children and presents four face morphs from a non-human-like

114 doll face to a completely human face (McLoughlin et al., 2018). We also created the Human

115 Silhouette scale that depicts the silhouettes of a human in a four-item scale with each item

116 showing a more complete cartoon silhouette of a human. We designed this scale to show the

117 "progress" toward being fully human without requiring children to need knowledge about

118 evolution that might be necessary to fully understand the Ascent of Man scale. Prior to the

119 examination of children, a validation study in adults was conducted to verify that the Face

120 Continuum scale and the Human Silhouette scale can capture dehumanizing responses as the

121 Ascent of Man scale does.

122

123 Power Analysis

124 To obtain $80 \%$ power to detect a small effect $(\mathrm{d}=0.25)$ with an alpha $=.05$ in the comparison

125 between ingroup and outgroup ratings of humanness on each scale using two-tailed Wilcoxon

126 signed rank tests, $\mathrm{G}^{*}$ Power specifies a sample size of 134. A priori power analysis for logistic

127 regressions specified a sample size of 110 to obtain $80 \%$ power to detect a medium effect (odds

128 ratio $=3.47$; see (Chen, Cohen, $\&$ Chen, 2010) in the comparison of dehumanization across

129 scales. To obtain $80 \%$ power to detect a medium effect (odds ratio $=3.47$ ) of a continuous

130 covariate (SDO, outgroup prosociality, and demographic variables for models in Study 1; SDO-

131 c, scale of humanness, social acceptance, outgroup sharing, outgroup punishment, and

132 demographic variables for models in Study 2) using logistic regressions, G*Power specifies a 
133 sample size of 55. Our final data included a sample size of 482 in adults and a sample size of 150

134 in children. The subsample size involved in analyses with continuous covariates is greater than

135 or equal to 58 . Therefore, we are sufficiently powered.

136

\section{Ethics Statement}

138 All research and consent procedures were approved by the campus institutional review boards at

139 Duke University (IRB Protocol Number: 2018-0035). For Study 2, all Zoo Atlanta granted

140 consent and an opt-out parental permission form was provided to all children's parents. All

141 studies reported in this paper comply with all relevant ethical regulations.

\section{Study 1}

144 We first validate that the three measures of humanness can elicit similar responses in adults

145 toward a group that is perceived as threatening in the US and was one of the most strongly

146 dehumanized group in previous research (Kteily et al., 2015). We systematically examine the

147 dehumanizing responses by observing the association with the endorsement of group-based

148 hierarchy and biased prosociality which were seen in previous investigations of dehumanization

149 (Kteily et al., 2015; Kteily et al., 2016).

151 Participants

152482 participants (mean age $=36.39, \mathrm{SD}=11.17 ; 267$ males, 215 females) completed an online

153 survey (see Appendix 1 for demographics of the participants and details of the survey). They

154 were recruited using Amazon's Mechanical Turk (MTurk: www.mturk.com) and completed the

155 survey as part of a larger study (see supplemental materials). All participants were from the 
156 United States and received $\$ 1$ for completing the survey regardless of their responses. Another

15726 participants did not complete the assessment based on predetermined demographic exclusion

158 criteria (i.e. they self-identified as belonging to the identity we used as outgroup. We assigned

159 "Muslims" as the outgroup because previous work with online samples shows U.S. participants

160 most heavily dehumanize this group relative to other racial and ethnic groups in this context;

161 Kteily et al, 2015).

162

163 Survey

164 Participants reported their racial or ethnic group and then responded to the survey which

165 included the following measures:

166 Humanness scales: allowed participants to rate their own ethnic group and Muslims using the

167 Ascent of Man, Face Continuum, and Human Silhouette. We code the responses on each scale

168 from $4=$ the least human-like to $1=$ fully human, so that higher scores represent lower perceived

169 humanness.

170 Social Dominance Orientation (SDO, Cronbach's $\alpha=0.95$ ): SDO was measured using the

171 standardized 16-item SDO 7 Scale (Ho et al., 2015). Participants rated their agreement with each

172 of the items following a 7 -point Likert scale (from $1=$ strongly disagree to $7=$ strongly agree).

173 Outgroup Prosociality: allowed participants to choose how to distribute a $\$ 0.5$ donation between

174 an ingroup and outgroup cause. The ingroup cause was a relief fund for victims of the Santa Fe

175 High School shooting. The outgroup cause was a relief fund for Afghanistan victims of war.

176 Participants were allowed to choose from six different amounts to donate to the Afghanistan fund

177 (from $\$ 0$ to $\$ 0.5$, interval $=\$ 0.1$ ). Whatever remained was distributed to the relief fund for the 178 ingroup. 
179 See Supplementary materials for measures of intergroup divide and demographic variables such

180 as sex, age, and political preference etc.

182 Analysis

183 Data were exported from Qualtrics in tabular form. Data were evaluated and one single response

184 for each participant was ensured using IP addresses and Mturk ID. R4.0.0 was used to analyze

185 the data. All three humanness measures were treated as ordinal. Cronbach's $\alpha$ coefficients of

186 ingroup and outgroup ratings were calculated respectively across the three humanness measures

187 to test for internal consistency. SDO, outgroup prosociality, and age were entered as continuous

188 variables. Sex was treated as binary. Wilcoxon signed rank tests were performed to demonstrate

189 blatant dehumanization by comparing the perceived humanness of the ingroup and the outgroup.

190 The effect size r's were calculated as Z/sqrt $\mathrm{N}$ and values were interpreted as small (0.1),

191 medium (0.3), and large (0.5) (Fritz, Morris, \& Richler, 2012). We calculated ordinal logistic

192 regressions with cumulative link mixed models to 1) compare dehumanization across the three

193 humanness measures, with Ascent of Man scale defined as the reference (See full results in

194 Supplementary Table 3); 2) test for a relationship between dehumanization and SDO as well as

195 outgroup prosociality (See full results in Supplementary Table 4-5); and 3) identify predictors of

196 dehumanization among demographic variables (See full results in Supplementary Table 10). All

197 tests were two-tailed, and p values were adjusted using Holm-Bonferroni method.

199 Results and discussion

200 Figure 2 presents participant responses on the three scales of humanness. Cronbach's $\alpha$

201 suggested acceptable internal consistency of the three scales on measuring outgroup humanness 
202 (Cronbach's $\alpha=.71$ ). Lower Cronbach's $\alpha$ (.43) was obtained across ingroup humanness

203 measures due to low variance of ratings on each scale (71.78\% of the participants rated the

204 ingroup as "fully human" on all three scales. See details of the distribution in Supplementary

205 Table 1).

206 Dehumanization of the outgroup is observed on each scale, with the outgroup more likely

207 rated as less human-like than the ingroup (Ascent of Man: $\mathrm{Z}=5.24$, effect size $r=.24, p<.001$;

208 Face Continuum: $Z=7.00$, effect size $r=.32, p<.001$; and Human Silhouette: $Z=5.16$, effect

209 size $\mathrm{r}=.24, \mathrm{p}<.001)$. No significant differences across the three humanness measures were

210 detected when comparing ratings of different groups, with the Face Continuum and Human

211 Silhouette eliciting similar levels of dehumanization as the Ascent of Man scale (see odds ratio

212 of the comparison of humanness ratings on each scale in Figure 3).

213 With ratings on the different scales being consistent with each other, we combined them

214 into a single measure of dehumanization by treating responses to the three scales as different

215 observations of the same variable. Using this combined measure, we find that participants who

216 were more likely to dehumanize the outgroup scored higher on SDO (odds ratio $=1.02,95 \% \mathrm{CI}$

$217=[1.01,1.04] ; \mathrm{Z}=3.70, \mathrm{p}<.001)$. Outgroup dehumanization is also associated with less

218 willingness to donate to the outgroup (odds ratio $=0.73,95 \% \mathrm{CI}=[0.61,0.86] ; \mathrm{Z}=-3.66, \mathrm{p}<$

$219.001)$. Together, the results validate the three humanness measures by showing that they elicit

220 similar dehumanizing responses in adults and replicating the link between dehumanization,

221 endorsement of social hierarchy and withdrawal of prosociality.

222

223 Study 2 
224 In Study 2, we examine dehumanization in children aged five years and older because they have

225 developed full-blown theory-of-mind ability and are able to perceive or deny a full human mind

226 of other individuals. We designed a situation in which participants compete against a

227 hypothetical peer group. We assessed responses toward the hypothetical outgroup using the three

228 humanness measures. To verify that participants understood the scales of humanness, a pre-test

229 was conducted. Similar to the observation of adults, we also tested the association of

230 dehumanization with SDO and empathic responses in children.

232 Participants

233 We tested 150 children ( $5-12$ years old, mean age $=7.72, \mathrm{SD}=1.64 ; 78$ boys, 72 girls).

234 Participants were tested in groups of similarly aged children (the age difference within a group

235 was always less than two years) at a week-long summer camp at Zoo Atlanta. The children were

236 from families of middle-class economic backgrounds and a relatively heterogenous racial

237 composition, but individual data was not collected on these variables to avoid possible

238 association of socioeconomic status or racial groups with dehumanizing representations. Each

239 child participated in the camp activities with the same group for 1-3 days immediately before the

240 experiment, so they were familiar with their group members. Each group was also assigned with

241 the name of a charismatic animal present at the zoo (i.e., the lions, flamingos, etc.), which

242 facilitated the sense of group identity. They came to the experiment in groups of 9-11 children

243 and remained with familiar members of their groups during the experiment - a knowledge

244 competition against a hypothetical peer group.

245

246 Procedure 
247 After entering the experimental room, children were directed to sit around a table. They could 248 see each other and the experimenter but could not see the responses other children made during

249 the experiment. They were asked not to speak with each other and to carefully follow the 250 experimenter's instruction. Children who were not able to follow the rules were allowed to finish 251 the experiment, but their groups data was discarded $(\mathrm{N}=7)$.

252 Children first participated in a comprehension pre-test (See Appendix 2 for the 253 procedure) that required they correctly order the different items of each of the three scales 254 according to the degree of humanness they illustrated (See Figure 1 for the correct order of 255 each). 139 (92.67\% of the sample) correctly ranked the scale items.

256 Children were next introduced by the experimenter to a hypothetical outgroup. The 257 experimenter told the children they would be competing against another group of campers from a 258 different zoo in a memory competition. The experimenter referred to this outgroup by their 259 group's animal name - the "Slug group". The campers were also told that the counselors at the 260 other camp thought the Slug group was "impolite, selfish and sloppy" (See Appendix 2 for the 261 standardized script). The experimenter then introduced the children to some animal facts and led 262 them in the memory competition that quizzed them about these same facts.

263 The children independently filled a physical paper-pencil survey by circling the answers 264 they agreed with the most (see Appendix 2 for the procedure and wordings). The experimenter 265 guided the children through the questions making sure all participants could complete them 266 regardless of their reading level. In doing so, experimenters never indicated how a child should 267 respond. The questionnaire included a measure of:

268 Humanness: Participants rated the humanness of their own group and the Slug group 269 using the Ascent of Man, Face Continuum, and Human Silhouette scales. This only required 
270 looking at the pictures of the scales and circling the item on the scale they believed best fit the

271 group they were evaluating. Identical to Study 1, we code the responses on each scale from $4=$

272 the least human-like to 1 = fully human.

273 Social Dominance Orientation-children (SDO-c, Cronbach's $\alpha=.66)$ : Participants

274 responded to four age appropriate questions using a 5-point Likert scale (from 1 = strongly

275 disagree to $5=$ strongly agree). These questions were adapted from the SDO scale (Ho et al.,

276 2015) and were designed to measure the endorsement of ingroup superiority over the Slug group.

277 Social Acceptance: The experimenter described a hypothetical scenario in which a

278 member of the Slug group joined the participants' group. The children were asked to indicate

279 their feelings about this person joining their group using a 7-point Likert scale (from $1=$ very

280 happy to $7=$ very sad).

281 Outgroup Punishment: This measure was added for the 2019 data collection season and

282 used with a subsample of 58 participants (mean age $=8.22, \mathrm{SD}=1.58 ; 30$ girls, 28 boys). The

283 experimenter described a hypothetical scenario in which a member of the Slug group betrayed a

284 non-Slug group member and tore apart the victim's painting. Participants were instructed to

285 decide how many hours the misbehaving person should be made to wait before she could visit

286 the zoo animals again. Children chose the number of hours from a list of choices (from 0h to 5h)

287 provided.

Outgroup Sharing: This measure was added in the latter half of the 2018 data collection

289 season and carried over into 2019. A subsample of 87 participants (mean age $=7.89, \mathrm{SD}=1.52$,

29043 girls, 44 boys) responded to it. Each participant was allowed to choose five of their favorite

291 stickers from a large collection. They were then asked how many they were willing to share with 
292 the Slug group. They did this by giving them back to the experimenter and marking on their 293 paper how many they would share.

294 Children then marked their age and gender on the survey and their responses were

295 collected. To protect the privacy of the children, no video recordings were made of the testing 296 sessions.

298 Analysis

299 Only test data from children who correctly ranked the scale items in the comprehension pretest 300 were included in the analysis ( $\mathrm{N}=11$ failed this pre-test). $\mathrm{R} 4.0 .0$ was used to analyze the data.

301 Humanness measures were treated as ordinal. Internal consistency of the three scales was

302 measured using Cronbach's $\alpha$. SDO-c, social acceptance, outgroup punishment, outgroup

303 sharing, and age were entered as continuous variables. Gender was treated as binary. Wilcoxon

304 signed rank tests were performed to demonstrate blatant dehumanization in children by

305 comparing the perceived humanness of the ingroup and the outgroup. Ordinal logistic

306 regressions were calculated using cumulative link mixed models to 1) compare dehumanization

307 across the three humanness measures, with Ascent of Man scale defined as the reference (See

308 full results in Supplementary Table 13);2) test the relation between dehumanization and SDO-c

309 as well as behavioral outcomes, including social acceptance, outgroup sharing and punishment

310 (See full results in Supplementary Table 14-S17); and 3) identify predictors of dehumanization

311 among age and gender (See full results in Supplementary Table 19). All tests were two-tailed, 312 and $\mathrm{p}$ values were adjusted using Holm-Bonferroni method.

\section{Results and discussion}


315 Figure 4 depicts the response of the children to each of the humanness scales. Cronbach's $\alpha$ 316 coefficients suggested relatively high internal consistency of the three scales on measuring 317 humanness of the outgroup $(\alpha=.83)$ and acceptable internal consistency on measuring ingroup

318 humanness $(\alpha=.69$, see details of the distribution in Supplementary Table 11).

319 Dehumanization is observed on each scale, with the outgroup being more likely to be

320 rated as less human-like than the ingroup (Ascent of Man: $\mathrm{Z}=8.52$, effect size $\mathrm{r}=.70, \mathrm{p}<.001$;

321 Face Continuum: $\mathrm{Z}=8.80$, effect size $\mathrm{r}=.72$, $\mathrm{p}<.001$; and Human Silhouette: $\mathrm{Z}=8.87$, effect

322 size $\mathrm{r}=.72, \mathrm{p}<.001)$. Children were more likely to dehumanize the outgroup on Human

323 Silhouette than the Ascent of Man scale (odds ratio $=3.25,95 \% \mathrm{CI}=[1.28,8.22] ; \mathrm{Z}=2.48, \mathrm{p}=$

324 .04). We did not see significant difference between ratings on Ascent of Man and Face

325 Continuum (see odds ratio of the comparison of humanness ratings on each scale in Figure 5).

326 Identical to Study 1, since responses to the three scales are consistent (based on

327 Cronbach's $\alpha$ ), we combined them and created a single measure of dehumanization. Using this

328 combined measure, results show that children who are more likely to dehumanize the outgroup

329 scored higher on SDO-c (odds ratio $=1.24,95 \% \mathrm{CI}=[1.12,1.38] ; \mathrm{Z}=3.99, \mathrm{p}<.001)$. The

330 dehumanization tendency is also associated with more willingness to punish the outgroup

331 member (odds ratio $=2.95,95 \% \mathrm{CI}=[1.90,4.57] ; \mathrm{Z}=4.83, \mathrm{p}<.001$ ), but only a trend for

332 children to show reduced willingness to share stickers with the outgroup (odds ratio $=0.73,95 \%$

$333 \mathrm{CI}=[0.54,0.97] ; \mathrm{Z}=-2.14, \mathrm{p}=.06)$. Ordinal logistic regressions reveal that neither age nor

334 gender predicted levels of dehumanization.

335

336 General discussion 
337 Results support the Early Emergence hypothesis. Children show higher levels of dehumanization

338 of outgroup than ingroup in response to all three representations of humanness. This pattern does

339 not change with age in middle childhood. Responses in adults and children were both associated

340 with participants' social dominance orientation - replicating the previously established pattern in

341 adults (Kteily et al., 2015), In both age groups, higher outgroup dehumanization was associated

342 with stronger agreement with statements of group dominance and inequality. Similar to the

343 previously demonstrated link between acceptance of punishment and outgroup dehumanization

344 in adults (Bandura et al., 1975; Jardina \& Piston, 2016), children who dehumanized more also

345 recommended more severe punishment for a misbehaving member of the Slug group. The link

346 between dehumanization and prosociality was also investigated. In adults, similar to previous

347 findings (Kteily et al., 2015), smaller allocations of support toward an outgroup charity was

348 associated with dehumanization of the outgroup. However, in children, only a non-significant

349 trend was observed between outgroup dehumanization and less sharing of stickers with the Slug

350 group. As well, we did not see a link between dehumanization and decreased social acceptance.

351 This suggests that dehumanization and disliking (i.e. the basis for prejudice) might already be

352 distinguishable early in development (Dore, Hoffman, Lillard, \& Trawalter, 2014).

353 There was only limited support for the Social Artifact hypothesis. As predicted by the

354 SAH, children did show a significant difference in the degree they dehumanized between the

355 scales. Children dehumanize more in response to the Human Silhouette scale than the Ascent of

356 Man scale. This might be because this simplified scale was more intuitive that might illicit a

357 stronger response once exposed to the erroneous belief it represents human progress. This might

358 be viewed as support for the idea that adolescent or adult levels of exposure are needed to fully

359 shape dehumanizing responses. However, the consistent responses across childhood suggest that 
360 dehumanization emerges before the prosociality toward outgroups become sensitive to social

361 norms (House et al., 2013). The overall similarity in the pattern of results between adults and

362 children in response to all three scales, despite this one difference, also suggests that

363 dehumanization does not require a lifetime of intense exposure to cultural norms or education to

364 be blatantly expressed. Instead, individual differences in the expression of dehumanization may

365 be largely due to socialization during infancy and early childhood.

366 We introduced a number of new instruments and results that provide initial support for

367 the validity of our new measures. Our results with adults replicate the pattern seen in previous

368 investigations of dehumanization using similar samples and questionnaires. With the three

369 humanness measures, we again observe the link between dehumanization, perception of social

370 hierarchy, and prosociality. Our pre-tests with the children helped demonstrate that the majority

371 of participants can intuitively understand all three scales with little, if any, explicit training. The

372 similarity of children's responses to the adult patterns also suggests these scales can measure

373 dehumanization at younger ages.

374 The main limitations of our tests derive from the methodological differences inherent in

375 comparing adults to preliterate children. Group psychology can be manipulated in adults simply

376 by mentioning the identity of a familiar outgroup. Questionnaires can also be completed

377 anonymously. With children, we felt the most age appropriate method was to use a manipulation

378 of group membership guided by an experimenter. The high level of outgroup dehumanization in

379 children might be attributable to this method of manipulation and lack of anonymity. For the

380 most meaningful quantitative comparisons across ages, future studies will need to develop more

381 comparable methods. 
Our findings have important implications for cognitive models and efforts to encourage

383 intergroup tolerance. The early appearance of dehumanization and concurrent moral

384 disengagement are coherent with the hypothesis that dehumanization occurs through a

385 specialized cognitive process that dampens theory-of-mind and denies a fully human mind to 386 others (Hare, 2017; Hare \& Woods, 2020). Future work can test this prediction further by

387 examining the link between theory-of-mind and dehumanization during early development.

388 Measures of metaphorical reasoning can also be used to directly test its role. In addition,

389 increases in self-control during adolescent development may also help explain individual

390 differences in blatant dehumanization (i.e. increases in self-control are likely linked to reduced

391 dehumanization; Hare, 2017). It will also be critical to test if positive intergroup contact reduces

392 or prevents dehumanization in adults and children (Bohman \& Miklikowska, 2020; Hodson,

393 Crisp, Meleady, \& Earle, 2018; Vezzali, Hewstone, Capozza, Trifiletti, \& Bernardo, 2017). Our

394 results seem to suggest early socialization by parents and exposure to a diverse range of people

395 in infancy and early childhood will have the greatest impact on reducing dehumanizing

396 tendencies. Essential to any of this future work will be the use of as diverse and representative as

397 possible. Previous research has revealed the prevalence of dehumanization across Western and

398 Middle Eastern countries (Kteily \& Bruneau, 2017), but few investigations have been conducted

399 with either East Asian or non-WEIRD societies. Advanced statistical tools can be leveraged to

400 allow for powerful quantitative comparisons across cultures and regions (Muthukrishna et al.,

401 2020). This type of research promises to reveal ways to further promote concern and cooperation 402 among human groups of all identities. 


\section{References}

405 Bain, P., Vaes, J., Kashima, Y., Haslam, N., \& Guan, Y. (2012). Folk conceptions of humanness: Beliefs about distinctive and core human characteristics in Australia, Italy, and China. Journal of cross-cultural psychology, 43(1), 53-58.

408

Bandura, A. (2016). Moral disengagement: How people do harm and live with themselves: Worth Publishers.

Bandura, A., Underwood, B., \& Fromson, M. E. (1975). Disinhibition of aggression through diffusion of responsibility and dehumanization of victims. Journal of Research in Personality, 9(4), 253-269.

413 Bedny, M., Pascual-Leone, A., \& Saxe, R. R. (2009). Growing up blind does not change the neural bases of Theory of Mind. Proceedings of the National Academy of Sciences,

Bohman, A., \& Miklikowska, M. (2020). Does classroom diversity improve intergroup relations? Short- and long-term effects of classroom diversity for cross-ethnic friendships and antiimmigrant attitudes in adolescence. Group Processes \& Intergroup Relations. doi:https://doi.org/10.1177/1368430220941592

Bruneau, E., Jacoby, N., Kteily, N., \& Saxe, R. (2018). Denying humanity: The distinct neural correlates of blatant dehumanization. Journal of Experimental Psychology: General,

423 Chas, A., Betancor, V., Delgado, N., \& Rodríguez-Pérez, A. (2018). Children consider their own 424 group to be more human than other social groups: Evidence from indirect and direct 425 measures. Social Psychology, 49(3), 125. 
426 Chen, H., Cohen, P., \& Chen, S. (2010). How big is a big odds ratio? Interpreting the magnitudes 427 of odds ratios in epidemiological studies. Communications in Statistics—simulation and $428 \quad$ Computation $($, 39(4), 860-864.

429 Cikara, M., Bruneau, E. G., \& Saxe, R. R. (2011). Us and them: Intergroup failures of empathy. $430 \quad$ Current Directions in Psychological Science, 20(3), 149-153.

431 Cikara, M., Eberhardt, J. L., \& Fiske, S. T. (2011). From agents to objects: Sexist attitudes and 432 neural responses to sexualized targets. Journal of cognitive neuroscience, 23(3), 540-551.

433 Cloutier, J., Ambady, N., Meagher, T., \& Gabrieli, J. (2012). The neural substrates of person 434 435 perception: Spontaneous use of financial and moral status knowledge. Neuropsychologia,

436 Costello, K., \& Hodson, G. (2014). Explaining dehumanization among children: The interspecies 437 model of prejudice. British Journal of Social Psychology, 53(1), 175-197.

438 Dhont, K., Hodson, G., Loughnan, S., \& Amiot, C. E. (2019). Rethinking human-animal 439 relations: The critical role of social psychology. Group Process \& Intergroup Relations,

Farrow, T. F., Jones, S. C., Kaylor-Hughes, C. J., Wilkinson, I. D., Woodruff, P. W., Hunter, M. 22(6), 769-784.

Dore, R. A., Hoffman, K. M., Lillard, A. S., \& Trawalter, S. (2014). Children's racial bias in perceptions of others' pain. British Journal of Developmental Psychology, 32(2), 218-

Fritz, C. O., Morris, P. E., \& Richler, J. J. (2012). Effect size estimates: current use, calculations, and interpretation. Journal of Experimental Psychology: General, 141(1), 2-18. 
Goff, P. A., Eberhardt, J. L., Williams, M. J., \& Jackson, M. C. (2008). Not yet human: implicit knowledge, historical dehumanization, and contemporary consequences. Journal of Personality and Social Psychology, 94(2), 292-306.

Harris, L. T., \& Fiske, S. T. (2006). Dehumanizing the lowest of the low: Neuroimaging responses to extreme out-groups. Psychological science, 17(10), 847-853.

Haslam, N., \& Loughman, S. (2014). Dehumanization and infrahumanization. Annu Rev Psychol, 65, 399-423. doi:10.1146/annurev-psych-010213-115045

Ho, A. K., Sidanius, J., Kteily, N., Sheehy-Skeffington, J., Pratto, F., Henkel, K. E., .. . Stewart, A. L. (2015). The nature of social dominance orientation: Theorizing and measuring preferences for intergroup inequality using the new $\mathrm{SDO}_{7}$ scale. Journal of Personality and Social Psychology, 109(6), 1003-1028.

Hodson, G., Crisp, R. J., Meleady, R., \& Earle, M. (2018). Intergroup contact as an agent of cognitive liberalization. Perspectives on Psychological Science, 13(5), 523-548.

House, B. R., Silk, J. B., Henrich, J., Barrett, H. C., Scelza, B. A., Boyette, A. H., . . Laurence, S. (2013). Ontogeny of prosocial behavior across diverse societies. Proceedings of the National Academy of Sciences, 110(36), 14586-14591.

Jack, A. I., Dawson, A. J., \& Norr, M. E. (2013). Seeing human: distinct and overlapping neural signatures associated with two forms of dehumanization. Neuroimage, 79, 313-328. doi:10.1016/j.neuroimage.2013.04.109

Jardina, A., \& Piston, S. (2016). Dehumanization of black people motivates white support for punitive criminal justice policies. Paper presented at the Annual Meeting of the American Political Science Association,, Philadelphia, PA. 
471 Kteily, N., \& Bruneau, E. (2017). Darker demons of our nature: The need to (re) focus attention on blatant forms of dehumanization. Current Directions in Psychological Science, 26(6),

473 487-494.

474 Kteily, N., Bruneau, E., Waytz, A., \& Cotterill, S. (2015). The ascent of man: Theoretical and 475 empirical evidence for blatant dehumanization. J Pers Soc Psychol, 109(5), 901-931. $476 \quad$ doi:10.1037/pspp0000048

477 Kteily, N., Hodson, G., \& Bruneau, E. (2016). They see us as less than human:

478 Metadehumanization predicts intergroup conflict via reciprocal dehumanization. Journal 479 of Personality and Social Psychology, 110(3), 343.

480 Levy, J., Goldstein, A., Influs, M., Masalha, S., Zagoory-Sharon, O., \& Feldman, R. (2016).

$481 \quad$ Adolescents growing up amidst intractable conflict attenuate brain response to pain of 482 outgroup. Proceedings of the National Academy of Sciences, 113(48), 13696-13701.

483 Markowitz, D. M., \& Slovic, P. (2020). Social, psychological, and demographic characteristics 484 485 of dehumanization toward immigrants. Proceedings of the National Academy of Sciences,

486 Martin, J., Bennett, M., \& Murray, W. S. (2008). A developmental study of the infrahumanization hypothesis. British Journal of Developmental Psychology, 26(2), 153162.

489 McCallum, C. (2001). Gender and sociality in Amazonia: how real people are made. Oxford: $490 \quad$ Berg.

491 McLoughlin, N., Tipper, S. P., \& Over, H. (2018). Young children perceive less humanness in 492 outgroup faces. Developmental science, 21(2), e12539. 
493 Muthukrishna, M., Bell, A. V., Henrich, J., Curtin, C. M., Gedranovich, A., McInerney, J., \&

494 Thue, B. (2020). Beyond western, educated, industrial, rich, and democratic (WEIRD)

495 psychology: measuring and mapping scales of cultural and psychological distance.

496 Psychological science, 31(6), 678-701.

497 Over, H. (2020). Seven challenges for the dehumanization hypothesis. Perspectives on

$498 \quad$ Psychological Science. doi:https://doi.org/10.1177/1745691620902133

499 Richins, M. T., Barreto, M., Karl, A., \& Lawrence, N. (2019). Empathic responses are reduced to 500 competitive but not non-competitive outgroups. Social neuroscience, 14(3), 345-358.

501 Rodríguez-Pérez, A., Betancor-Rodríguez, V., Ariño-Mateo, E., Demoulin, S., \& Leyens, J.-P.

502 (2014). Normative data for 148 Spanish emotional words in terms of attributions of

503 humanity.[Datos normativos de 148 términos afectivos en dimensiones relacionadas con

504 la atribución de humanidad]. Anales de Psicología/Annals of Psychology, 30(3), $1137-$

$505 \quad 1145$.

506 Rodríguez, V. B., Villar, A. C., Rodríguez-Pérez, A., \& Rodríguez, N. D. (2016).

507 Infrahumanization in children: An evaluation of 70 terms relating to humanity.

$508 \quad$ Psicothema, 28(1), 53-58.

509 Smith, D. L. (2020). On inhumanity: Dehumanization and how to resist it: Oxford University $510 \quad$ Press.

511 Sumner, W. G. (1906). Folkways: A study of the sociological importance of usages, manners, 512 customs, mores, and morals. Boston: Ginn.

513 Trounson, J. S., Critchley, C., \& Pfeifer, J. E. (2015). Australian attitudes toward asylum 514 seekers: Roles of dehumanization and social dominance theory. Social Behavior and 515 Personality: an international journal, 43(10), 1641-1655. 
516 van Noorden, T. H., Haselager, G. J., Cillessen, A. H., \& Bukowski, W. M. (2014).

517 Dehumanization in children: The link with moral disengagement in bullying and $518 \quad$ victimization. Aggressive behavior, 40(4), 320-328.

519 Vezzali, L., Hewstone, M., Capozza, D., Trifiletti, E., \& Bernardo, G. A. D. (2017). Improving 520 intergroup relations with extended contact among young children: Mediation by 521 intergroup empathy and moderation by direct intergroup contact. Journal of Community $522 \quad \&$ Applied Social Psychology, 27(1), 35-49.

523 Viki, G. T., Osgood, D., \& Phillips, S. (2013). Dehumanization and self-reported proclivity to 524 torture prisoners of war. Journal of Experimental Social Psychology, 49(3), 325-328.

525 Wilks, M., Caviola, L., Kahane, G., \& Bloom, P. (2020). Children prioritize humans over 526 animals less than adults do. doi: https://doi.org/10.1177/0956797620960398 


\section{Author contributions}

528 Both authors contributed to the study design. W.Z. performed testing, data collection, data

529 analysis and interpretation under the supervision of B.H. Both authors wrote the manuscript and 530 approved the final version of the manuscript for submission.

531

\section{Acknowledgements}

533 We thank the Zoo Atlanta for hosting our research, to the parents and children for participating 534 and to Aleah Bowie, Erica Blair, Feruth Kidane, Julia Villegas, and Morgan Ferrans who helped 535 collect the data. We thank Philip White and Zachary White who gave us advice on statistics. We 536 also wish to thank Aleah Bowie, Michael Tomasello, Sarah Gaither and Ashley Jardina for

537 invaluable comments they provided on an earlier version of the manuscript. Funding for this 538 work was provided by Duke University. 


\section{$540 \quad$ Figures}
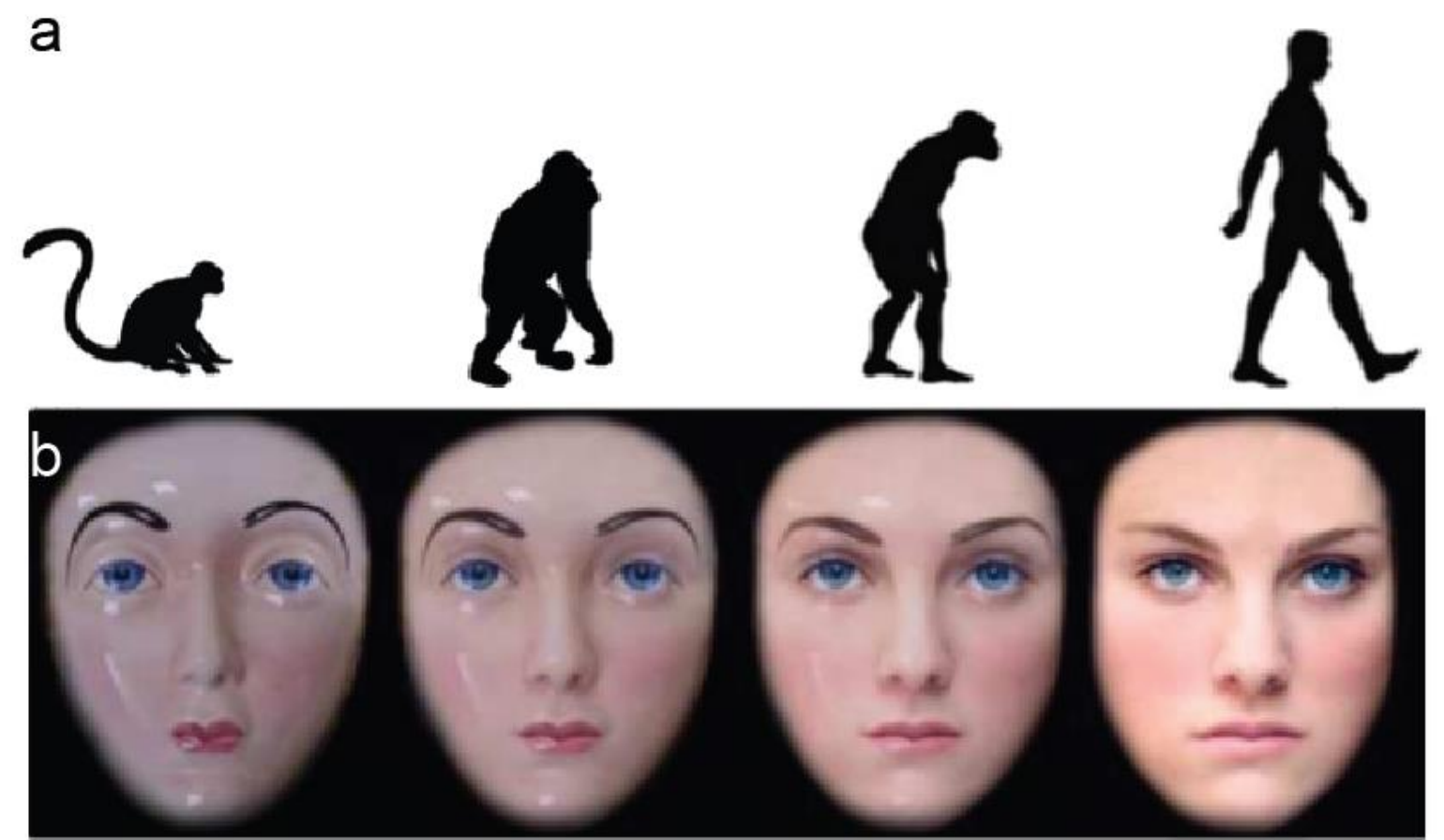

C

Not human A little bit human A medium amount human Completely human

541 4 3
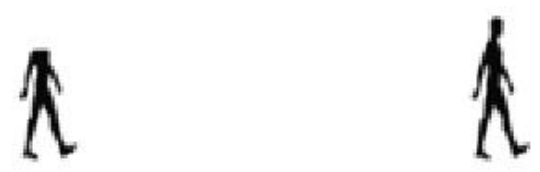

d

IV

2

1

542 Figure 1. The three measures of humanness. a) Ascent of Man, b) the Face Continuum and c)

543 the Human Silhouette. Ratings on each measure were coded following the 4-point scale from 1=

544 fully human to 4 = the least human-like (as illustrated in Panel d).

545

546

547

548 


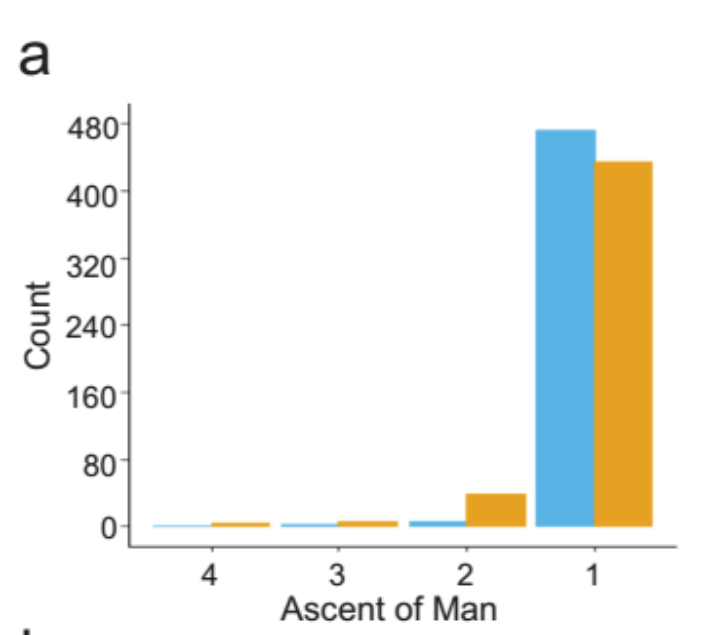

Target

Ingroup

Outgroup

b
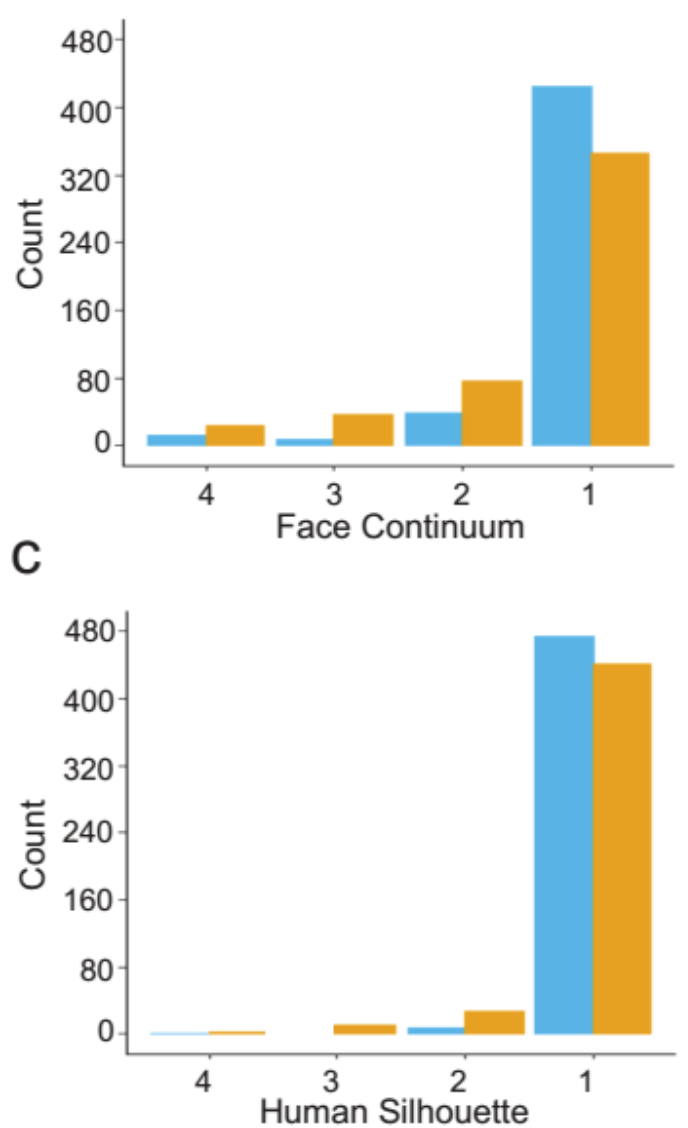

550 Figure 2. Perceived humanness of the ingroup and the outgroup across three measures in adults

551 (Study 1). The ratings were coded following the 4-point scale from $1=$ fully human to $4=$ the

552 least human-like. As such, higher ratings represent lower perceived humanness. Each histogram

553 demonstrates the number of participants who made the rating. 


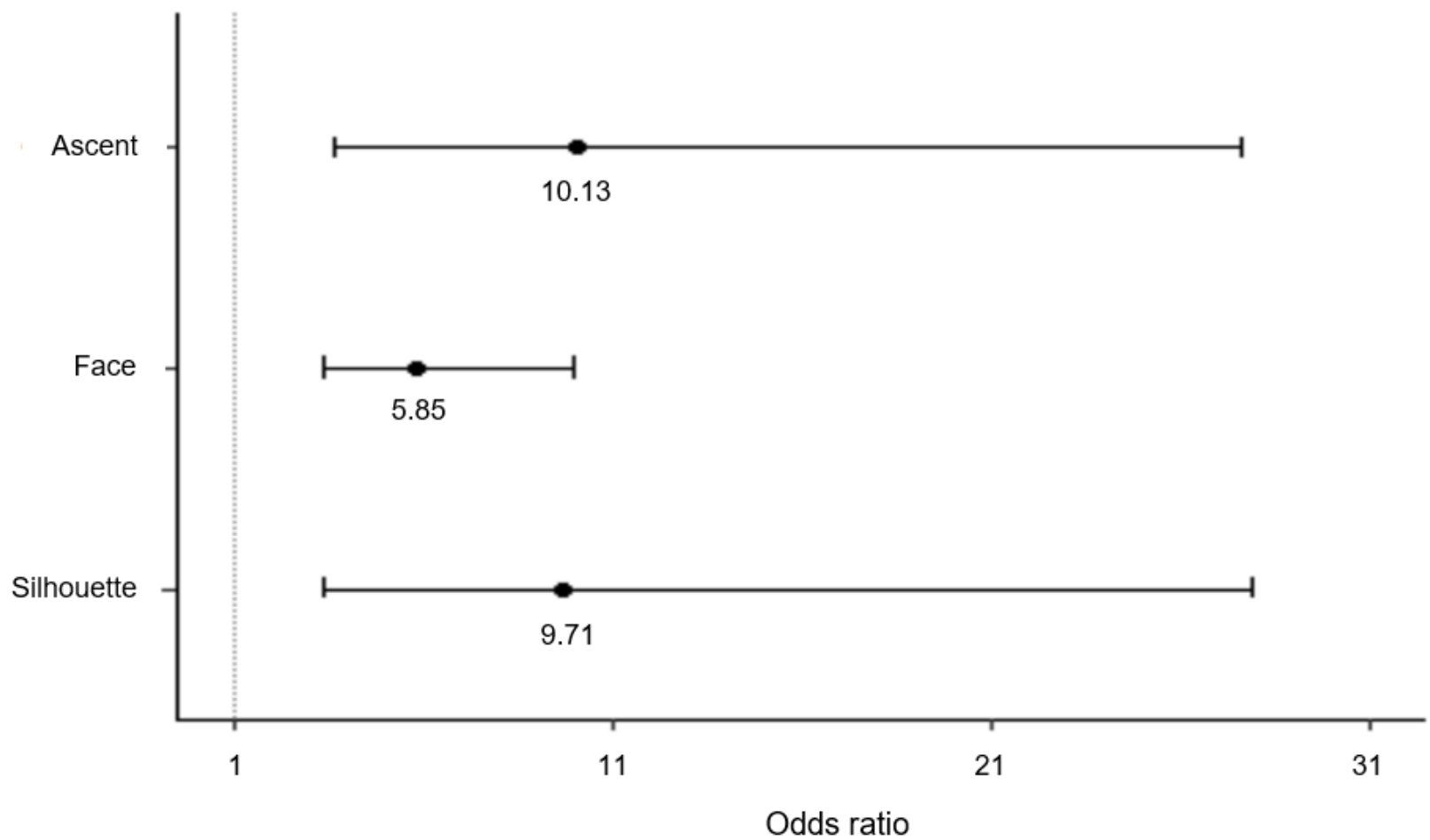

556 Figure 3. Dehumanization on each measure of humanness in adults (Study 1). Dehumanization

557 is defined that the outgroup as compared to the ingroup is more likely (odds ratio $>1$ ) to be rated

558 less human-like. Ascent $=$ Ascent of Man. Face $=$ The Face Continuum. Silhouette $=$ The Human

559 Silhouette. Error bars represent 95\% confidence intervals. Odds ratios and confidence intervals

560 are calculated from the results reported in Supplementary Table 3. 

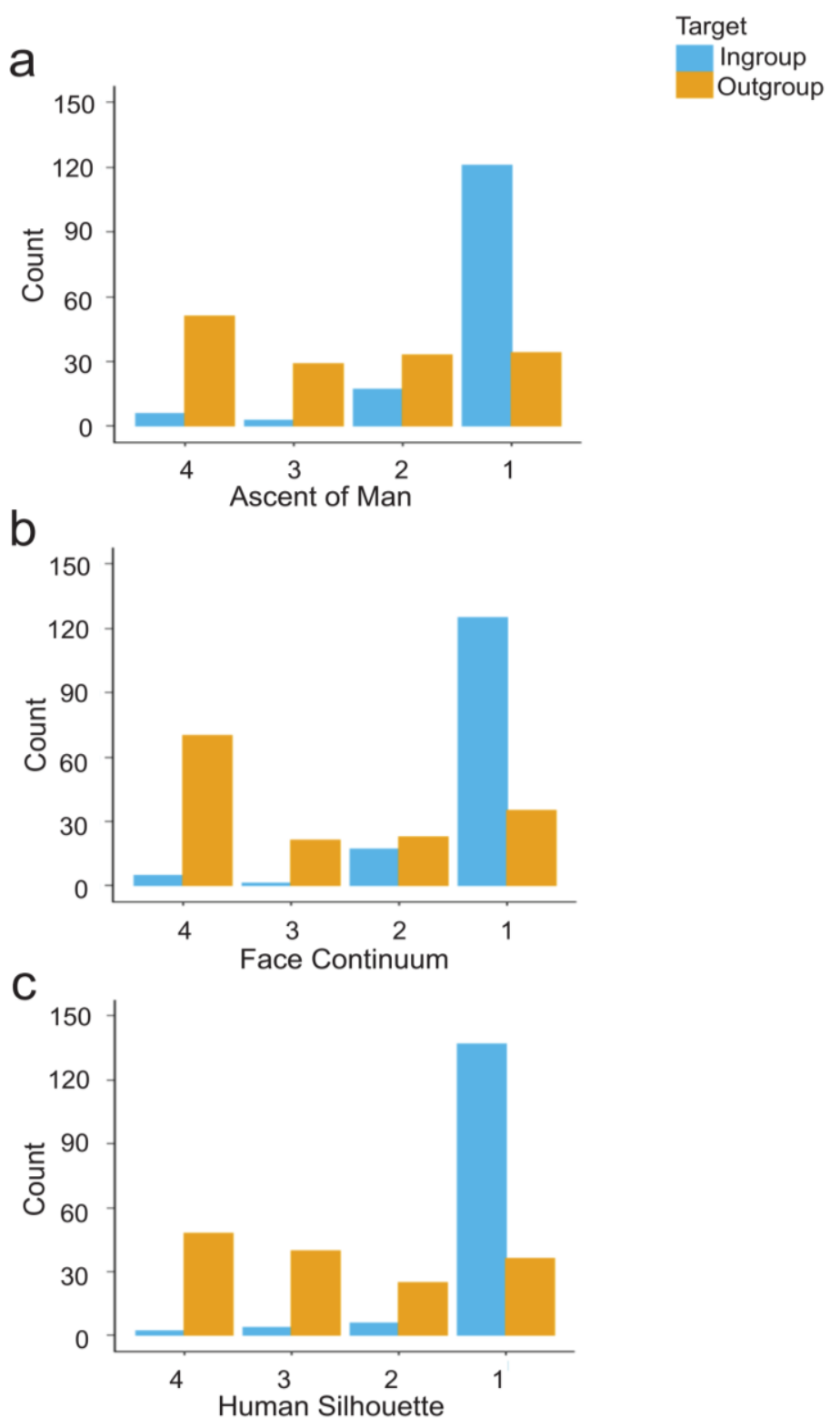

562 Figure 4. Perceived humanness of the ingroup and the outgroup across three measures in

563 children (Study 2). The ratings were coded following the 4-point scale from $1=$ fully human to 4

$564=$ the least human-like. As such, higher ratings represent lower perceived humanness. Each

565 histogram demonstrates the number of participants who made the rating. 


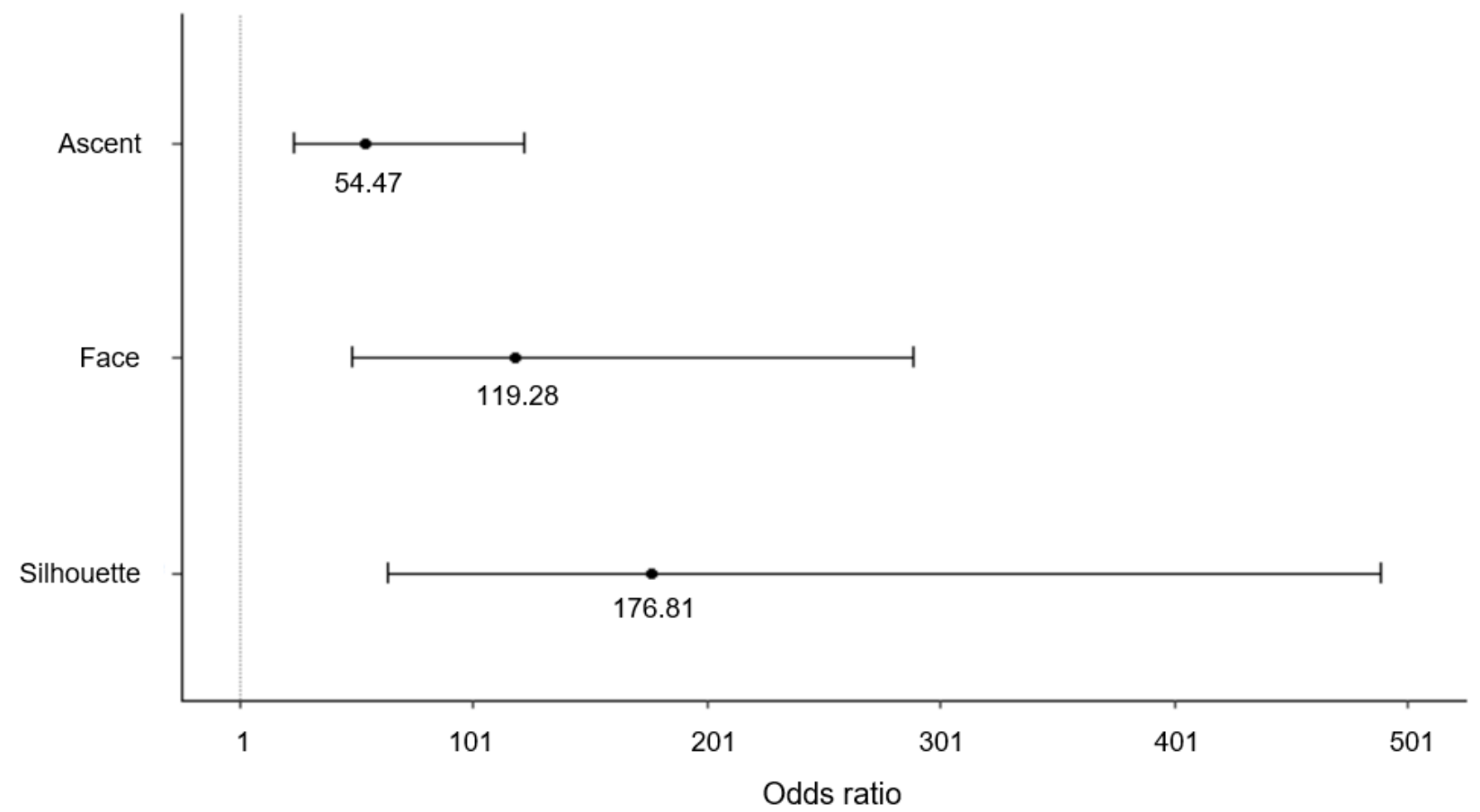

568 Figure 5. Dehumanization on each measure of humanness in children (Study 2).

569 Dehumanization is defined that the outgroup as compared to the ingroup is more likely (odds

570 ratio $>1$ on the horizontal axis) to be rated less human-like. Ascent $=$ Ascent of Man. Face $=$ The

571 Face Continuum. Silhouette $=$ The Human Silhouette. Error bars represent $95 \%$ confidence

572 intervals. Odds ratios and confidence intervals are calculated from the results reported in

573 Supplementary Table 13.

574 